\title{
Design and Implementation of Real-Time Monitoring GIS Terminal Based on Android System
}

\author{
Haoliang $\mathrm{Li}^{1, \text { a }}$, Haobo $\mathrm{Li}^{2, \mathrm{~b}}$, Mingyuan Yang ${ }^{3, \mathrm{c}}$ and Na Wang ${ }^{4, \mathrm{~d}}$ \\ Zhengzhou University, School of Information Engineering, 450001 Zhengzhou, China

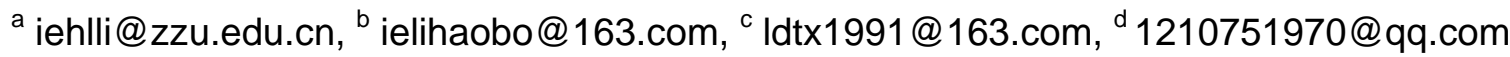

Keywords: GIS, GPS, Real-Time Monitoring System, Android Application.

\begin{abstract}
Real-Time Monitoring System(RTMS) is the result of the development of information technology, which has made a huge difference to the life and work. The Android based GIS terminal is designed and implemented for RTMS, integrated with Internet communication, GPS, and intelligent navigation technology. The GPS module is used to collect positioning information of the terminal. Utilizing the cloud computing, the design gives a real-time route guidance to improve efficiency of traditional GPS navigation. With the Internet technology, this paper could complete geographic information uploading function. Based on Android application development technology, the paper designs the terminal software to complete monitoring function. By using the Android software designed in this paper, the GIS terminal can realize positioning and navigation features, upload monitoring information to the back-end system. And the test result proves that the design of the application development can meet the expected functional requirements of the RTMS.
\end{abstract}

\section{Introduction}

Since the RTMS plays a very important role in logistics or other related industry [1], it's greatly necessary to design a monitoring GIS terminal. With the development of embedded device and mobile communication technology, the portable intellectual equipment can be applied to various industries as a monitoring terminal, especially Android based intelligent devices [2]. This paper proposes and designs an Android based intelligent terminal for the RTMS, which provides comprehensive functions, such as GPS positioning, intelligent navigation, information uploading etc. The Android is a free open-source system, and the design of Android application is easier than other system. Meanwhile, the cost of the terminal designing and development is inexpensive, and the development cycle is relatively short. Therefore, the Android based monitoring GIS terminal make it possible for large-scale applying in the monitoring system.

Based on Android application, the terminal provides many function modules [3], such as real-time positioning, route navigation, mobile communication. All the functions, as is proposed above, depend on the wireless Internet technology, using the Android network development skills.

In the design of the GIS terminal, Android software application has been completed to realize key functions of the terminal. The main content of this paper can be summarized as the method of terminal implementation in three parts: GPS positioning, intelligent navigation, and monitoring information uploading. And after terminal testing, the result shows that it can achieve the expected functional aims of the real-time monitoring system.

\section{Design and Implication}

Based on the requirement of the RTMS, that requires collecting the device's geographic location and other real-time information, the GIS terminal has been designed with three main functions. Utilizing GPS, cloud computing, and mobile communication technology, it can flawlessly complete required information uploading task.

To obtain position information, the GIS terminal uses GPS module of the Android device [4]. The Android software has been designed to realize map position service. In this paper, Baidu Map is used to develop software application. The software could use Baidu Map to get device's location 
information, through the GPS module integrated with the terminal. In the Android software application developing project, using Baidu Map to position current location information, and a service is designed to transmit that information to the monitoring system. The design flow of positioning function is shown in Fig. 1.

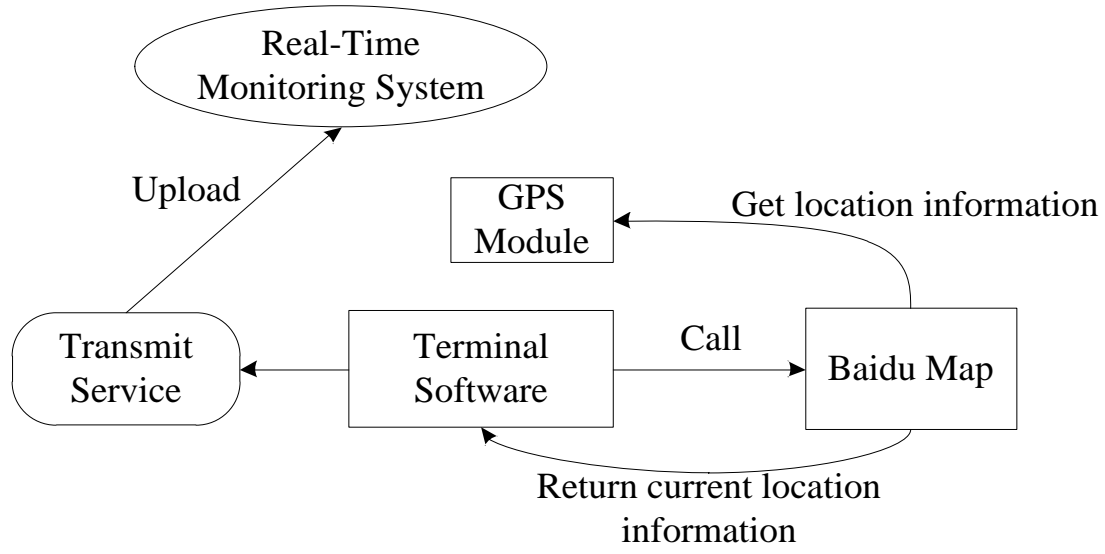

Fig. 1 Position function designing flow.

The RTMS requires GIS terminals' location information to realize monitor function, and also needs other information, such as vehicle state information, delivery information etc. With the comprehensive information, the RTMS can realize real-time management to every single terminal. To complete the aim, this paper designs a data transmitting method based on Android network development skills.

Using the Internet to communicate with monitoring system, the application should have the permission to access Internet. So before design the communication method, the permission must be declared in AndroidManifest file of the project. After finishing the preparatory work, a data transmitting method is designed in the software project, which could package location information, device working condition and other required information of the system to the Http request [5]. And with mobile communication technology, the terminal can upload various related information to the monitoring system. And the system can decode the Http request to obtain monitoring information. In the condition of terminal working environment, that the network communication probably under the 3G or 4G network, a method is design to judge the way to access network, and switch network connection parameters. Fig. 2 shows the flowchart of network communication design.

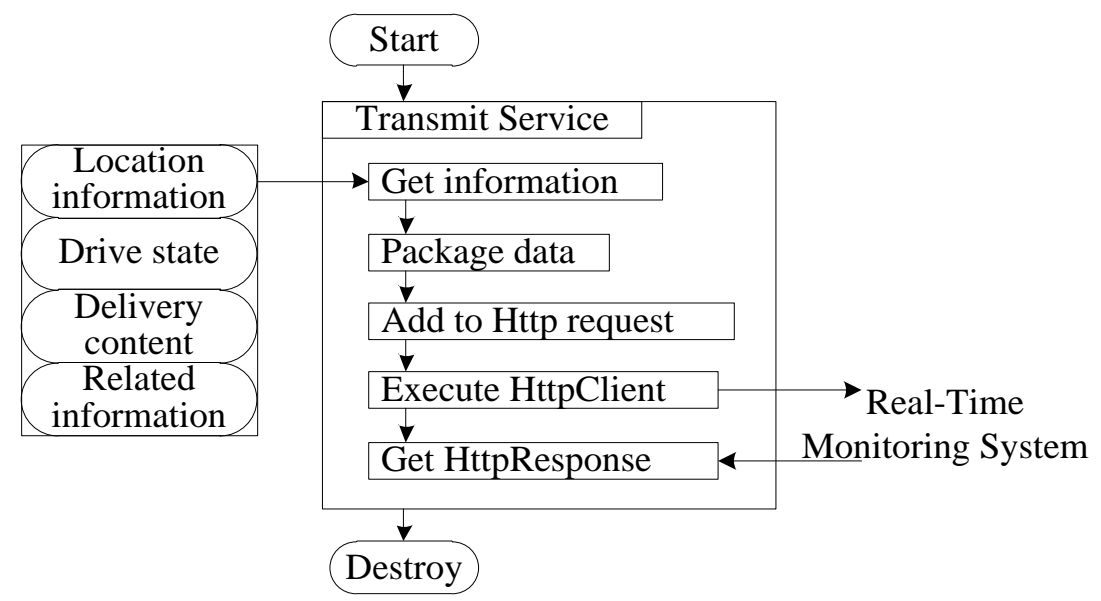

Fig. 2 The flowchart of network communication design.

The paper designs intelligent navigation feature for the terminal, using the cloud computing technology and traditional GPS navigation [6]. The intelligent navigation function mainly bases on cloud computing of the monitoring system. In the design of terminal software, it applies the Baidu Map navigation service to calculate the shortest route, according Map guidance to travel. The main part of the intelligent navigation is that the terminal software can receive monitoring system order to 
change route planning. The monitoring system can analyze the real-time route traffic condition, and send warning order to the terminal, which nears to the traffic congested road. According the warning order, the terminal can recalculate navigation plan under the condition of avoiding congestion. In the program of the terminal software, a background service is designed to monitor the warning order, and after received the order the software would call Notification of Android system to remind user to change current navigation strategy. Fig. 3 shows the mechanism of intelligent navigation function.

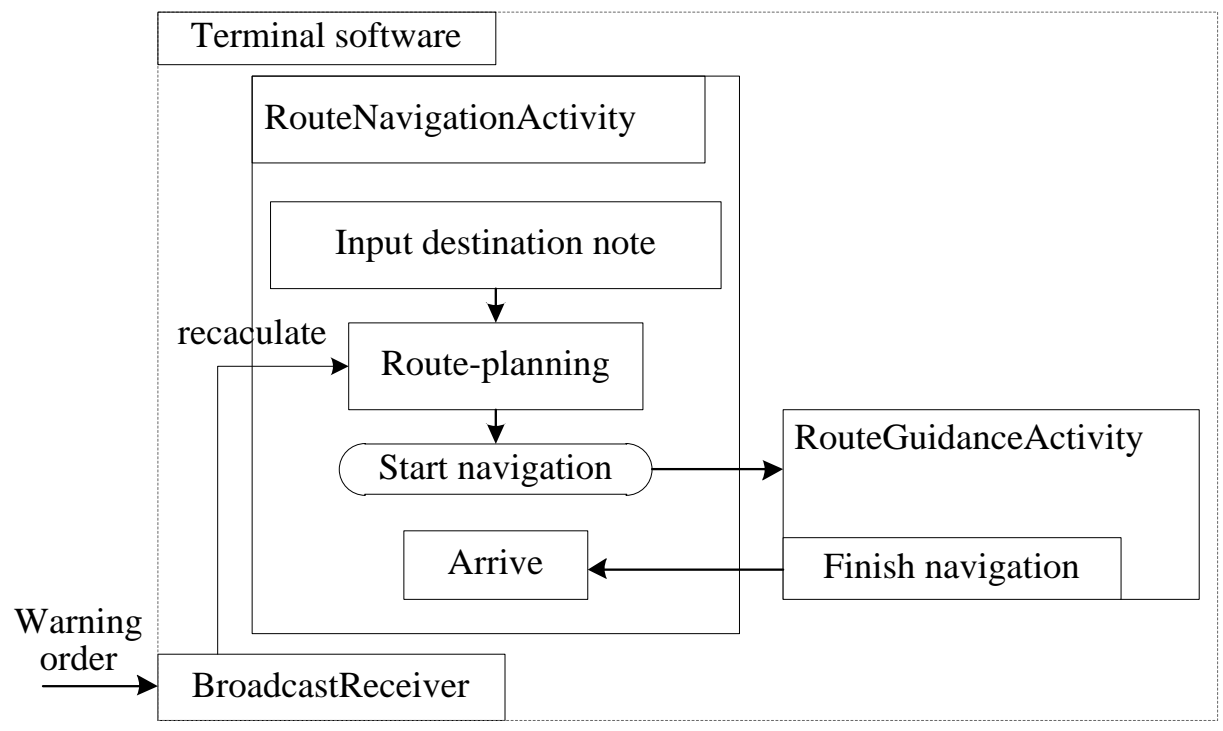

Fig. 3 The mechanism of intelligent navigation function.

By designing the intelligent navigation mechanism, it can improve the efficiency of delivery process and reduce the waste of vehicle fuel. With the unique function, the terminal can achieve the aims of reducing costs of industry.

\section{Test Results and Discussion}

The GIS terminal, designed in this paper, relies on Android software application, integrated with GPS, mobile communication, and intelligent navigation technology. And the terminal functions have been tested under the real-time monitoring system [7]. Results shows that it can satisfy the requirement of the system, which can correctly provide monitoring information uploading and intelligent navigation functions. The test results have been shown in Fig. 4 and Fig. 5.

In the Fig. 4, the navigation feature has been tested. It shows that the terminal provides shortest route guidance plan for the vehicle, under the condition of avoiding traffic congestion. And the route plan could randomly change, following the warning order of the monitoring system.

In the Fig. 5, it shows the monitoring information in the back-end system, transmitting by the

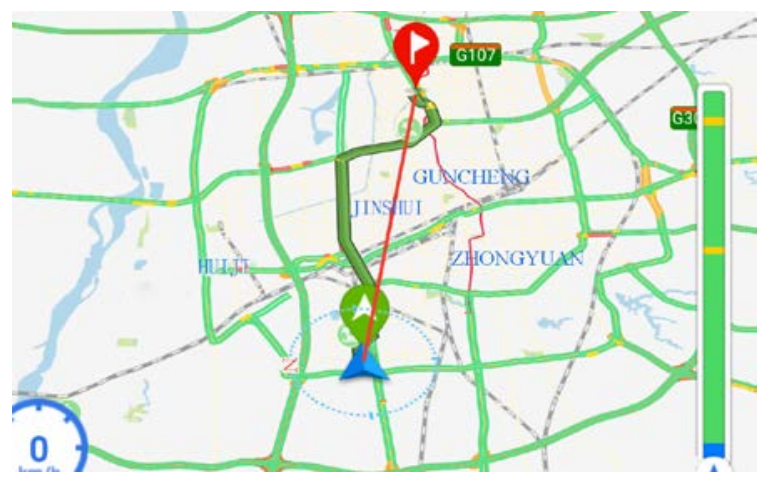

Fig. 4 Navigation function of the terminal

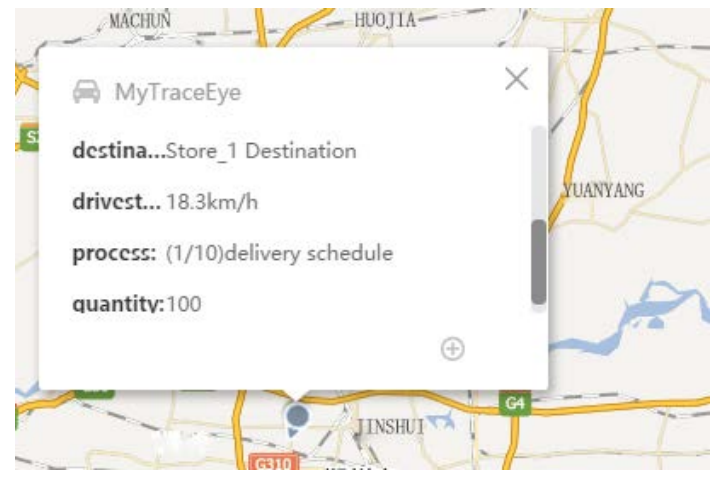

Fig. 5 Monitoring information of the terminal

terminal software. As is shown that the terminal location information and other related information have been unloaded to the monitoring system. 


\section{Conclusions}

The GIS terminal is designed for the purpose of monitoring every single part in the system, and providing optimized guidance. With the GPS, mobile communication, and cloud computing technology, the paper successfully completed the GIS terminal design for the real-time monitoring system. By Android software program designing, the terminal can provide multi-functions for the user, which includes location positioning, intelligent navigation, and monitoring information transmitting. And the test result shows that it can perfectly finish the task design in the software application. The terminal bases on Android system, which means that more functions can be added to complement its tools. Therefore, the Android based GIS terminal will have a good prospect in the future in various of industry.

\section{References}

[1] P. Jeefoo: International Conference on Information Science and Applications (2014), p.1-3.

[2] Arora P K, Bhatia R, Parkash S, et al: International Conference on Reliability, INFOCOM Technologies and Optimization (2015), p.1-5.

[3] Tung S C, Li W J, Huang S M: Applied Mechanics and Materials Vols. 284-287 (2013), p. 3211-3215.

[4] E. D'Andrea and F. Marcelloni: IEEE International Conference on Smart Computing (SMARTCOMP) (2016), p.1-5.

[5] K. Yamamoto and T. Ikeda, IEEE 18th International Conference on High Performance Computing and Communications; IEEE 14th International Conference on Smart City; IEEE 2nd International Conference on Data Science and Systems (HPCC/SmartCity/DSS) (2016), p.50-57.

[6] S. Exauty, H. Gao, X. Wang and X. Yu, International Conference on Logistics, Informatics and Service Sciences (LISS) (2016), p.1-4.

[7] Information on http://lbsyun.baidu.com/trace/admin/service 